\title{
Author Correction: Pathogenic bone marrow B cells
}

Nicholas J. Bernard

Nature Reviews Rheumatology (2019) https://doi.org/10.1038/s41584-019-0166-1

Published online 9 January 2019

In the originally published online version of this article there was an error in the page number of the reference to the original article published in Nature Communications, and the associated link was therefore incorrect. The page number was listed as 5217 and should have been 5127. This error has now been corrected in the HTML and PDF versions of the manuscript.

https://doi.org/10.1038/s41584-019-0167-0 I Published online 14 January 2019

\section{Author Correction: Pathogenesis and therapeutic interventions for ANCA-associated vasculitis}

\author{
Daigo Nakazawa, Sakiko Masuda, Utano Tomaru and Akihiro Ishizu(D)
}

Nature Reviews Rheumatology (2019) https://doi.org/10.1038/s41584-018-0145-y

Published online 12 December 2018

In the originally published online version of this article there were errors in the Supplementary Information. All three Supplementary Tables had incorrectly numbered references. These errors have now been corrected in the HTML and PDF versions of the manuscript.

https://doi.org/10.1038/s41584-019-0168-z I Published online 17 January 2019 